\title{
ESTRATÉGIAS DE SOBREVIVÊNCIA DE INVERTEBRADOS TERRESTRES EM FLORESTAS INUNDÁVEIS DA AMAZÔNIA CENTRAL: UMA RESPOSTA À INUNDAÇÃO DE LONGO PERÍODO
}

\author{
Joachim ADIS ${ }^{1}$
}

\begin{abstract}
RESUMO - As florestas inundáveis na Amazônia Central e suas margens adjacentes sofrem alagaçòes de vários metros de altura por 5-7 meses cada ano, devido ao pulso monomodal de inundação dos rios. Os invertebrados terrestres adaptaram-se à este ecossistema, envolvendo várias estratégias de sobrevivência. A fauna compõe-se de animais terricolas e arboricolas. Ambos os grupos incluem não migrantes e migrantes. A reação migratória dos animais terricolas é horizontal (à frente da linha d'água), vertical (subida temporária para troncos ou copas de árvores) ou inclui um vôo temporário para florestas de terra firme. Não migrantes têm estágios ativos ou dormentes abaixo d'água. Os dormentes passam a inundaçào em abrigos disponiveis em condições naturais, em abrigos fabricados ou em estágio de ovo. Animais arborícolas não migrantes vivem e se reproduzem exclusivamente em troncos e copas das árvores, enquanto que os migrantes tem estágios de desenvolvimento os quais vivem também no chão. São dadas as características e exemplos de espécies para cada uma destas categorias. $\mathrm{O}$ pulso de inundação é considerado como determinante original da migração. Entretanto, a maioria dos invertebrados se tornaram sensiveis aos ecofatores secundários, na sua maioria abióticos. Somente em algumas espécies, a migração ainda é diretamente relacionada com o pulso de inundação.
\end{abstract}

Palavras chaves: Pulso de inundação, florestas inundáveis, migrações, invertebrados terrestres, estratégias de sobrevivência, Amazônia.

Survival Strategies of Terrestrial Invertebrates in Central Amazonian inundation Forests; a Response to Long-Term Flooding.

ABSTRACT - Inundation forests in Central Amazonia and their adjacent shores are covered by several meters of flood water for 5-7 months each year, due to the monomodal periodic flood pulse of rivers. Terrestrial invertebrates have adapted to this ecosystem by evolving several survival strategies. The fauna comprises both, terricolous and arboricolous animals. Both groups include non-migrants and migrants. Migratory reaction of terricolous animals is horizontal (following the high-water line), vertical (temporal ascent to trunk or canopy of trees) or includes a temporal flight to upland forests. Non-migrants have active or dormant stages under water. The latter pass inundation in naturally available retreats, in self-made retreats or as eggs. Non-migrant arboricolous animals reproduce and live exclusively in the trunk and canopy region, whereas migrants include life stages that live on the ground as well. Characteristics and examples of species are given for each of these categories. The flood pulse is regarded as the original determinant of migration. However, most invertebrates have become sensitive to secondary, mainly abiotic factors. Only in a few species is migration still directly related to the cycle of flooding.

Key Words: flood pulse, inundation forests, migration, terrestrial invertebrates, survival strategies, Amazon.

\section{INTRODUÇÃO}

Estratégias de sobrevivência sâo "adaptações de organismos às condições externas desfavoráveis, que aumentam sua capacidade de sobrevivência"(TISCHLER, 1984). Estratégias são "padrões de comportamento determinados geneticamente, os quais surgem através de seleção natural" (SOUTHWOOD,

Instituto Max-Planck para Limnologia, Grupo de Trabalho em Ecologia Tropical, Caixa

Postal 165, D-24302 Plön, Alemanha, em cooperação com o Instituto Nacional de

Pesquisas da Amazônia (INPA), Manaus/AM, Brasil. 
1977, 1988). Isto não implica que haja uma "ação consciente" (finalista) do organismo, e os termos "padrão" e "opção" podem ser substituidos por "estratégia" (CHAPLEAU et al., 1988).

Os invertebrados terrestres em sistemas periodicamente inundados necessitam de "estratégias de sobrevivência" especiais. O desenvolvimento destas estratégias é determinado pelo tipo de inundação (definida matematicamente como oscilação). Este é definido pelo número de inundações (= frequência), sua altitude (= elongação ou amplitude máxima) e a duração da inundação no ciclo anual (= fase aquática). Estas características representam o assim chamado "pulso de inundação" (JUNK et al., 1989). Este é o "mecanismo regulador primário" ou "ecofator primário" (SCHAEFER \& TISCHLER, 1983) no sistema.

Na Amazônia Central, o pulso de inundação é monomodal (Fig. 1). Uma mudança periódica anual no nível das águas do rio Solimões-Amazonas e do rio Negro atinge em média $9.8 \mathrm{~m}$, de acordo com as leituras do nivel da água de 1903 a 1989 no porto de Manaus. As florestas perto dos rios, então chamadas florestas inundáveis e suas margens adjacentes (localizadas entre os rios e as florestas inundáveis, como p.ex. "praias") sofrem inundações que se extendem por muitos metros, com uma duração entre cinco e sete meses cada ano (Março/ Abril - Agosto/Setembro), dependendo da elevação da terra e da altura da inundação anual (Fig. 1; cf. ADIS, 1981; JUNK, 1989). Acredita-se que este processo venha se repetindo por milhões de anos (ADIS, 1984). Através deste longo período de adaptação sazonal ao ecossistema, animais e plantas foram capazes de se ajustar à inundação regular e periódica (cf. IRMLER, 1981; JUNK, 1989; JUNK et al., 1989; WORBES, 1986, 1989; WALKER, 1992). Ao longo de muitos outros rios, como o Reno, a freqüência, a amplitude e o período de oscilação do pulso de inundação são variáveis (Fig. 1), portanto, não previsíveis, ocorrendo freqüentemente condições catastróficas (DISTER, 1985, 1988).

Neste trabalho resume-se 20 anos de pesquisa sobre as estratégias de sobrevivência de invertebrados terrestres, que têm sido determinadas através dos estudos ecológicos na região inundável da Amazônia Central nos arredores de Manaus nos últimos 20 anos (cf. ADIS, 1992a, 1997; BECK, 1983).

\section{ESTRATÉGIAS}

A fauna de invertebrados terrestres da Amazônia Central compõe-se de animais terrícolas e arboricolas. Os animais do chão ou animais do solo (cf. ADIS 1981, 1992a) são em sua maioria habitantes noturnos da camada orgânica e da parte superior da camada inorgânica do solo (e.g. ADIS \& RIBEIRO, 1989). Os animais arborícolas ou animais "da árvore" vivem na região dos troncos e das copas das árvores (e.g. ADIS et al., 1984). Ambos os grupos incluem animais migrantes e não migrantes. Os não migrantes completam seu ciclo de vida em apenas um habitat, seja no chão ou nas árvores. Entretanto, 

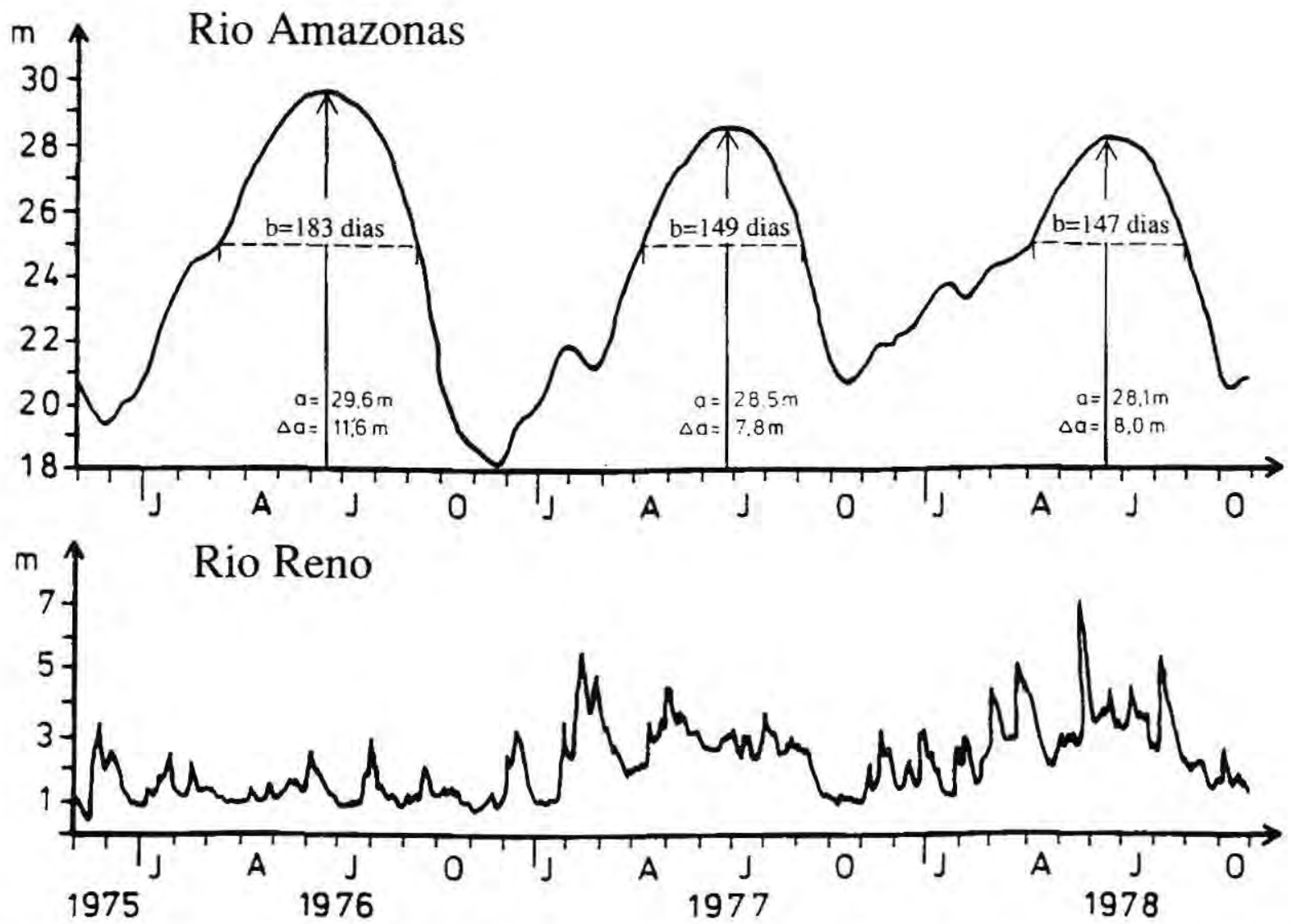

Figura 1. Niveis mensais da água (Novembro 1975-1978) do rio Amazonas nas cercanias de Manaus (Brasil) e do rio Reno nas cercanias de Worms (Alemanha; dados de acordo com JUNK, 1989). A altura da inundação anual (a) assim como as diferenças entre o menor e o maior nivel da água ( $\Delta$ a) são fornecidas para o rio Amazonas. A duração da enchente nas florestas inundáveis a uma altitude de $25 \mathrm{~m}$ nas cercanias de Manaus é indicada para os respecti os anos (b).(Para maiores explicaçōes consultar o texto). 
movimento de pequena escala dentro do habitat e ocorrências temporárias em outros habitats são possíveis, Os migrantes se reproduzem primariamente em somente um dos habitats, mas seu ciclo de vida inclui estágios que mudam periodicamente de habitat ou biótopo.

\section{Animais terrícolas: migrantes}

Três modos de ação são distinguidos, os quais representam reações dos animais do solo à inundação sazonal (Fig. 2):

\section{a) Migração horizontal}

Este tipo de migração é efetuada por animais que se movem em direção à terra firme (regiões altas não inundáveis) à frente da linha d'água, que avança durante os períodos de subida de nivel dos rios. Eles retornam para a floresta inundável com o recuo das águas.

As espécies de animais que se utilizam desta estratégia são incluídas na categoria 1 da figura 2 .

\section{b) Migração vertical}

Os animais que utilizam este tipo de migração movem-se temporariamente para 0 tronco das àrvores, podendo passar a fase aquática no próprio tronco ou nas copas. Suas caracteristicas são:

Todos ou somente um dos estágios ativos da espécie inicia a subida ao tronco das árvores um pouco antes da inundação ou com algumas semanas de antecedência, com o início da estação chuvosa.

A principal reprodução ocorre no chão da floresta durante a fase terrestre.

O ciclo de reprodução e a duração do estágio de vida são sincronizados com à flutuação periódica do nivel da água.

As espécies de animais que se utilizam desta estratégia são incluídas na categoria 2 da figura 2 .

\section{c) Vôo}

Os animais capazes de voar se utilizam deste tipo de migração através vôos temporários para a floresta de terra firme vizinha, a qual não é inundável. Suas características são:

A saida da floresta de inundação usualmente ocorre algumas semanas antes da inundação; mas após o início da estação chuvosa.

A reprodução principal se efetua no chão da floresta durante a fase terrestre.

A duração do estágio de desenvolvimento terrestre e a velocidade do ciclo reprodutivo completo são sincronizados com a flutuação periódica do nivel da água.

As espécies de animais que se utilizam desta estratégia são incluídas na categoria 3 da figura 2 .

\section{Animais terrícolas: não migrantes}

Entre os animais do solo, não migrantes, ocorre um estágio que permanece submerso entre cinco e sete meses por ano. Faz-se uma distinção entre espécies que são ativas sob a água e aquelas que permanecem dormentes durante a fase aquática.

\section{a) Dormência sob a água}

O grupo de animais que não abandonam seu habitat e passam a fase aquática em dormência sobre ou dentro do solo da floresta inundável:

1) em abrigos disponíveis em 
condições naturais.

2) em abrigos fabricados.

3) em estágio de ovo.

As espécies de animais que se utilizam desta estratégia são incluídas nas categorias 4-6 da figura 2 .

\section{b) Ativos sob a água}

São aqueles animais que permanecem ativos no solo da floresta inundada ou nas regiōes de troncos que permanecem sob a água.

As espécies de animais que se utilizam desta estratégia sãoincluídas na categoria 7 da figura 2 .

\section{Animais arborícolas: migrantes}

Neste grupo são incluídos aqueles animais que vivem principalmente sobre os troncos ou nas copas das árvores onde realizam sua reprodução principal. Suas características são:

Durante a fase terrestre, estágios de desenvolvimento colonizam também o solo, onde ocorre uma reprodução secundária.

Todos ou somente alguns dos estágios no ciclo de vida fazem parte da migração descendente e ascendente nos troncos.

As espécies de animais que se utilizam desta estratégia são incluídas na categoria 8 da figura 2 .

\section{Animais arborícolas: não migrantes}

Este grupo compreende aqueles animais que vivem e se reproduzem exclusivamente nos troncos e nas copas das árvores, onde efetuam somente movimentos de pequena escala. Suas características são:

Durante a fase terrestre, estágios dos animais arborícolas aparecem somente por um período temporário no chão da floresta.

As espécies de animais que se utilizam desta estratégia são incluídas na categoria 9 da figura 2 .

\section{DISCUSSÃO}

De acordo com os resultados obtidos a nivel de espécie, o pulso de inundação pode ser considerado como o determinante principal da migração ascendente e descendente dos invertebrados terrestres nos troncos das árvores. Entretanto, este "mecanismo regulador primário" ou "ecofator primário" atua somente sobre algumas espécies (e.g. A.v. tarumanis (Oligochaeta), E. latus, A. parvus (Opiliones), C. taruma, Tr. amazonicus (Araneae) na figura 2). Muitos dos invertebrados terrestres aparentemente se tornaram sensíveis aos ecofatores secundários, principalmente abióticos, os quais já não são diretamente relacionados ao ciclo de inundação. A migração dos animais do chão para o tronco das árvores, assim como o vôo para a floresta de terra firme é acionada principalmente pela estação chuvosa (Dezembro - Maio; RIBEIRO \& ADIS, 1984), a qual se inicia três a quatro meses antes da inundação, e pelas mudanças nos fatores edáficos e climáticos que ela causa. Exemplos estão no aumento da umidade do solo e na umidade relativa do ar $(T$. amazonicus, $T$. migrans (Pseudoscorpiones)), assim como no decréscimo da temperatura máxima do ar e na diminuição da diferença entre a temperatura máxima e mínima do ar 


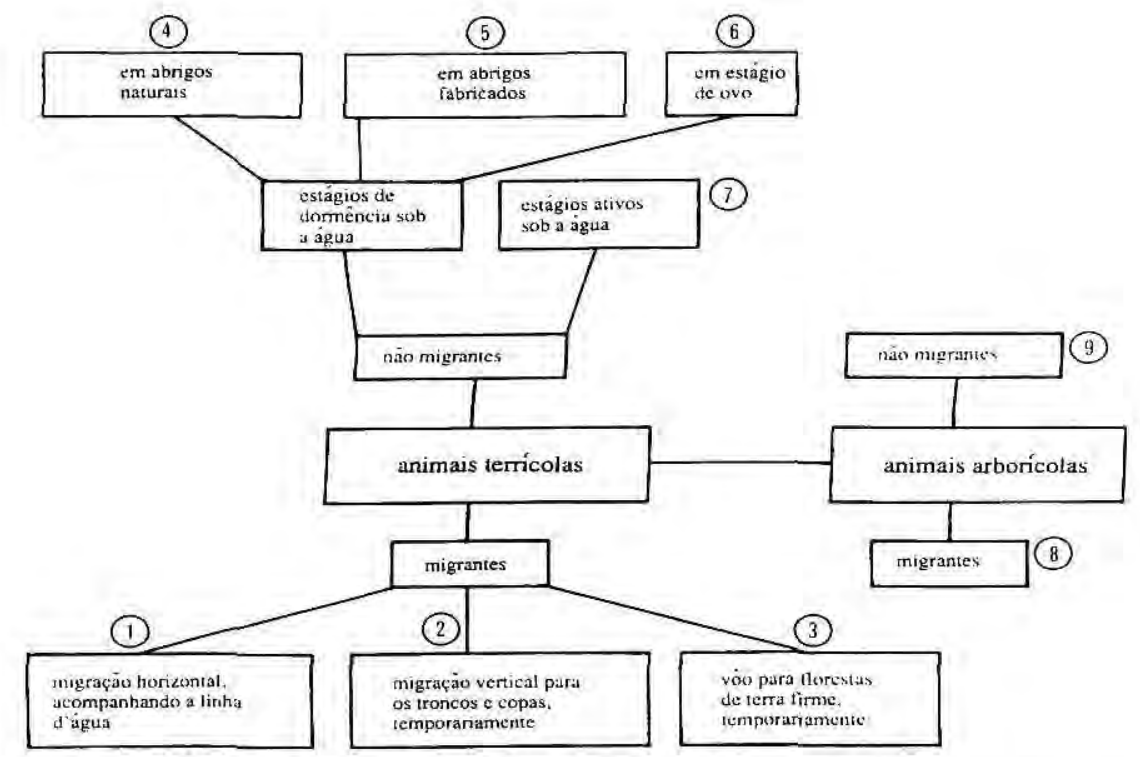

Figura 2. Estratégias de sobrevivēncia de invertebrados terrestres em regiỏes inundáveis ao longo

do rio Solimões-Amazonas e rio Negro na Amazónia Central, com exemplos das espécies estudadas (de acordo com ADIS, 1992b; complementado).

1 - a) Oligochaeta: Glossoscolecidae: Tairona tipema (ADIS \& RIGHI, 1989)

b) Coleoptera: Staphylinidae: Lathrohium sp. (IRMLER, 1979)

2 - a) Oligochaeta: Glossoscolecidae: Andiorthimus tenezulamus tarmananis (ADIS \& RIGHI, 1989)

b) Pseudoscorpiones: Chtoniidae: Thyrantocththonits amazonicus, Thyramochthonitrs migrans (ADIS \& MAHNERT, 1985)

c) Opiliones: Cosmetidae: Etccyortula lata: Gonyleptidac: Auranus parvis (= Sngnidius inflatus in FRIEBE \& ADIS, 1983)

d) Araneac; Gnaphosidae: Camilina taruma. Tricongitus amazonicus; Ochyroceratidae: Theotima mimutissima; Onopidae:

Xyccarph sp. (HÖFER, 1990; PLATNICK \& HÖFER, 1990)

e) Symphyla: Scutigerellidac; Hanseniella arhorea (ADIS \& SCHELLER, 1984; ADIS et al., 1996b)

f) Diplodat Paradoxomatidae: Mestosoma hylaeicum; Fuhrmannodesmidae: Cuttervodesmts adisi (ADIS, 1992e: ADIs et al., 1996a)

g) Archaeognatha: Meinertellidae: Neomachileltus scandens (ADIS \& STURM, 1987a,b; WOLF \& ADIS, 1992)

h) Isoptera: Termilidae: Anoplotermes spp. (MARTIUS, 1989, 1990)

i) Coleoptera: Cicindelidae: Pentacomia egregia (PAARMANN et at., 1982)

j) Coleoptera: Carabidae: Scarites sp. (ADIS et al., 1990)

k) Hymenoptera: Formicidae: Acromymex lundi carti (ADIS, 1982a)

3 - a) Coleoptera: Carabidae: Polyderis nvmphia (ADIS et al.1986)

4 - a) Symphyla: Scolopendrellidae: Ribautiella amazonica (no interior de raizes; ADIS, 1992b; ADIS er al., 1996b)

b) Coleoptera: Oedemeridae: Sisenopiras gounellei (no interior de pau morto; ADIS \& ARNETT, 1987)

5 - a) Diplura: Japygidae (casulo de seda; ADIS et al., 1989)

b) Homoptera: Pseudococcidae. Cicadidac (proteç̧ão de cera; ADIS \& MESSNER, 1991: MESSNER \& ADIS, 1992)

6-a) Acari: Eremobelbidae: Eremohetha foliata. Hypochthoniidae: Parhypochthonius sp. (BECK, 1972)

b) Chilopoda: Henicopidae: Lamyctes adtisi (ADIS, 1992b; ZALESSKAJA. 1994)

c) Collembola: Arthropleona \& Symphypleona (ADIS \& MESSNER, 1991)

7 - a) Acari: Haplozetidae: Rostrozetes foveolatus (BECK, 1969; MESSNER ot at.. 1992)

b) Diplopoda: Pyrgodesmidae: Gonographis adisi (ADIS, 1986; MESSNER \& ADIS, 1988)

8 - a) Pseudoscorpiones: Miratemnidae: Brasilatemnus trowni: Olpiidae: Pachyolpium irmgardae (ADIS \& MAHNERT, 1985; ADIS et al., 1988)

9 - a) Araneae: Pholcidac: Blechrocetis sp. (Hoper, 1990); Pisauridae: Trechalea amazonica $(=T$. manauensis em CARICO el at., 1985; cf. CARICO, 1993)

b) Diplopoda: Pseudonannolenidae: Epinannolene arhorea (ADIS, 1984)

c) Archaeognatha: Meinertellidae: Meinertellus adisi: Neoniachilellus adisi (ADIS \& STURM, 1987b)

d) Isoptera: Termitidae: Nasutitermes spp., Termes medioculanus (MARTIUS, 1989, 1992)

e) Coleoptera: Carabidae: Agya spp., Euchila spp., Miotachys spp., Moirainpa amazona (= Tachyina sp.; cf. ADIS, 1982b)

f) Hymenoptera: Formicidae: Cephatothes attatus, Daceton armigerum (ADIS, 1981) 
à altura do chão da floresta $(P$. nympha (Carabidae, Coleoptera); cf. Fig. 2). Efeitos macroclimáticos, causados pelo "El Niño", têm uma influência adicional (ADIS \& LATIF, 1996; ADIS et al. 1996a). Migrações verticais são observadas nas florestas de inundação, mas não na terra firme da Amazônia Central (ADIS \& SCHUBART, 1984).

A fauna de invertebrados terrestres das florestas inundáveis ao longo do rio Negro compreende muitas espécies endêmicas (cf. ADIS \& MAHNERT, 1990). Entretanto, esta fauna também deve incluir componentes que antigamente efetuaram uma migração secundária da região da margem adjacente à floresta inundável e da região de terra firme. Esta migração se deslocava em direção à floresta inundável. Tem sido mostrado que o desenvolvimento de ciclos específicos de reprodução pelos animais terrícolas e arborícolas é relacionado com a transição entre a terra firme e a floresta de inundação. Por exemplo, o pseudoescorpião Brazilatemnus browni, duas especies de Meinertellidae (Archaeognatha) e quatro espécies de Symphyla (Myriapoda) aparentemente são plurivoltinos na terra firme mas uniou bivoltinos nas florestas inundáveis (ADIS, 1992a; ADIS \& STURM, 1987b; ADIS et al., 1988, 1996b). A aquisição de uma periodicidade anual, principalmente como reação à inundação por um período de seis meses, também pode ser vista como um requerimento básico para a colonização de zonas temperadas. Um exemplo disto é encontrado na familia Carabidae (Coleoptera), cujo centro evolucionário é localizado nas regiões inundáveis ao longo dos rios tropicais (ERWIN 1979, 1981; ERWIN \& ADIS, 1982). Algumas espécies de animais imigrantes nas florestas inundáveis não diferem morfologicamente, mas elas diferem ecologicamente, fenologicamente assim como geneticamente daquelas das regiões de terra firme adjacentes, a um grau tão elevado, que podem representar novas espécies e subespécies (ADIS et al., 1988; ADIS \& STURM, 1987b; WOLF \& ADIS, 1990). Portanto, as florestas inundáveis da Amazônia devem contribuir para a diversidade de invertebrados neotropicais (cf. ADIS, 1990), tanto pelas espécies endêmicas encontradas (p.ex. em Pseudoscorpiones e Diplopoda), quanto pelos possiveis processos de especiação observados em imigrantes sob influência de inundações anuais durante um período de longo prazo (= pulso de taxon; cf. ERWIN \& ADIS, 1982). Porém, a plasticidade fenotípica (= estado morfológico, fisiológico e etológico em resposta à condições ambientais, conforme WEST-EBERHARD 1989) e a variabilidade genética têm que ser estudadas para cada espécie (cf. TOMIUK et al., 1996; WALKER, 1992). A floresta inundável, aparentemente, representa um ecossistema próprio. A fauna característica de invertebrados terrestres fornece evidências de que ela tornou-se na sua maioria independente, se comparado à região da margem do 
rio e da terra firme. A adaptação de invertebrados terrestres à floresta inundável é, geralmente, de natureza etológica e/ou fisiológica. Adaptações morfológicas são excepcionais (e.g. MESSNER \& ADIS, 1988). Em meio à pluralidade de habitats e recursos, a probabilidade para sobreviver dos invertebrados terrestres nas florestas inundáveis depende da diversidade das suas estratégias de sobrevivência, aludida como valência ecológica.

\section{AGRADECIMENTOS}

Sou grato a todos os colegas que de uma maneira ou outra contribuíram para este trabalho, especialmente a muitos taxônomos e aos cientistas do Grupo de Ecologia Tropical do Instituto Max-Planck para Limnologia (MPIL), em Plön (Alemanha), assim como do Instituto Nacional de Pesquisas da Amazônia (INPA), em Manaus (Brasil). Sinceros agradecimentos ao corpo técnico de Manaus e de Plön, pela ajuda valiosa recebida no campo e no laboratório. Ao Dr. Nigel Stork, do Museu de História Natu$\mathrm{ral}$, em Londres (Inglaterra), e às Dras. Elizabeth Franklin e Ilse Walker do INPA em Manaus (Brasil) como também à Sra. Vivian Bühler do MPIL em Plön (Alemanha) pelos valiosos comentários e ajuda recebida no manuscrito.

\section{Bibliografia citada}

ADIS, J. 1981. Comparative ecological studies of the terrestrial arthropod fauna in Central Amazonian inundaton forests. Amazoniana, 7(2): 87-173.

_. 1982a. Eco-Entomological observations from the Amazon: III. How do leafcutting ants of inundation forests survive flooding? Acta Amazonica,
12(4): 839-840.

-_- 1982b. Zur Besiedlung zentralamazonischer Überschwernmungswälder (Várzea-Gebiet) durch Carabiden (Coleoptera). Arch. Hydrobiol., 95(1/4): 3-15.

-... - 1984. 'Seasonal igapó'-forests of Central Amazonian black-water rivers and their terrestrial arthropod fauna. In: SIOLI H. (ed.) The Amazon. Limnology and landscape ecology of a mighty tropical river and its basin. Monographiae Biologicae, Junk Publ. Dordrecht. p245-268.

-.. - 1986. An „,aquatic" millipede from a Central Amazonian inundation forest. Oecologia, 68(3): 347-349.

-_. 1990. Thirty million arthropod species too many or too few? J. Tropic. Ecology, 6: 115-118.

-_. - 1992a. Überlebensstrategien terrestrischer Invertebraten in Überschwemmungswäldem Zentralamazoniens.Verh. naturwiss. Ver. Hamburg, 33(NF): 21-114,

-_. - 1992b. How to survive six months in a flooded soil: Strategies in Chilopoda and Symphyla from Central Amazonian floodplains. In: ADIS J, \& TANAKA S. (eds.) Symposium on life-history traits in tropical invertebrates. (INTECOL, Yokohama, Japan 1990). Studies on Neotropical Fauna and Environment, 27(2-3). Swets \& Zeitlinger. Lisse. pl17-129.

-_ 1992c. On the survival strategy of Mestosoma hylaeicum Jeekel (Paradoxomatidae, Polydesmida, Diplopoda), a millipede from Central Amazonian floodplains), Ber: Nat.med. Verein Innsbruck, Suppl. 10: 183-187.

-.. - 1997. Terrestrial invertebrates: Survival strategies, group spectrum, dominance and activity patterns. In: JUNK, W.J. (ed.) The Central Amazon floodplain: Ecology of a pulsing system. Ecological Studies, Springer. Heidelberg. No prelo.

ADIS, J.; ARNETT JR, R.H. 1987. Eco-Entomological observations from the Amazon: VI. Notes on the natural history and flood resistance of Sisenopiras gounellei Pic (Coleoptera: Oedemeridae). Coleopterists Bulletin, 41(2): 171-172. 
ADIS, J.; LATIF, M. 1996. Amazonian arthropods respond to El Niño. Biotropica, 28(3): 403-408

ADIS, J.; MAHNERT, V. 1985. On the natural history and ecology of Pseudoscorpiones (Arachnida) from an Amazonian black-water inundation forest. Amazoniana, 9(3): 297-314.

ADIS, J.; MAHNERT, V. 1990 . On the species composition of Pseudoscorpiones from Amazonian dryland forests and inundation forests in Brazil. Revue suisse Zool., 97(1): 49-53.

ADIS, J.; MESSNER, B. 1991. LangzeitÜberflutungsresistenz als Überlebensstrategie bei terrestrischen Arthropoden. Beispiele aus zentralamazonischen Überschwemmungsgebieten. Dtsch. ent. Z. $N F, 38(1-3): 211-223$.

ADIS, J.; RIBEIRO, M.O, 1989. Impact of deforestation on soil invertebrates from Central Amazonian inundation forests and their survival strategies to long-term flooding. Water Quality Bulletin, 14(2); $88-98+104$.

ADIS, J.; RIGHI, G. 1989. Mass migration and life cycle adaptation - a survival strategy of terrestrial earthworms in Central Amazonian inundation forests. Amazoniana, 11(1): 23-30.

ADIS, J.; SCHELLER, U. 1984. On the natural history and ecology of Hanseniella arborea Scheller (Myriapoda, Symphyla, Scutigerellidae), a migrating symphylan from an Amazonian black-water inundation forest. Pedobiologia, 27(1): 35-41.

ADIS, J.; SCHUBART, H.O.R. 1984. Ecological research on arthropods in Central Amazonian forest ecosystems with recommendations for study procedures, In: COOLEY J.H.; GOLLEY F.B. (eds.) Trends in ecological research for the 1980s. NATO Conference Series, Series I: Ecology, Plenum Press. New York, London. pl11-144.

ADIS, J; STURM, H. 1987a. Flood-resistance of eggs and life-cycle adaption, a survival strategy of Neomachilellus scandens (Meinertellidae, Archaeognatha) in Central Amazonian inundation forests. Insect
Sci. Applic:, 8(4-6): 523-528.

ADIS, J,; STURM, H. 1987b. On the natural history and ecology of Meinertellidae (Archaeognatha, Insecta) from dryland and inundation forests of Central Amazonia. Amazoniana, 10(2):197-218.

ADIS, J.; GOLOVATCH, S.; HAMANN, S. 1996a. Survival-strategy of the terricolous millipede Cutervodesmus adisi (Fuhrmannodesmidae, Polydesmida) in a blackwater inundation forest of Central Amazonia (Brazil) in response to the flood pulse. In: GEOFFROY, J.-J., MAURIÉS, J.-P.; NGUYEN DUYJACQUEMIN, M. (eds.) Acta Myriapodologica. Mém. Mus. natn. Hist. nat. 169: 523-532.

ADIS, J; LUBIN, Y.D; MONTGOMERY, G.G. 1984. Arthropods from the canopy of inundated and terra firme forests near Manaus, Brazil, with critical considerations on the pyrethrumfogging technique. Stud. Neotrop. Fauna Environ., 19(4): 223-236.

ADIS, J.; MAHNERT, V.; MORAIS, J.W. DE; RODRIGUES, J.M.G. 1988.Adaptation of an Amazonian pseudoscorpion (Arachnida) from dryland forests to inundation forests. Ecology, 69(1): 287-291.

ADIS, J.; MESSNER, G.; GROTH, I. 1989. Zur Überflutungsresistenz und zum Spinnvermögen von Japygiden (Diplura). Zool. Jb. Anat., 119(3/4): 371-382.

ADIS, J; MORAIS, J.W. DE; SCHELLER, U. 1996b. On abundance, phenology and natural history of Symphyla from a mixedwater inundation forest in Central Amazonia, Brazil. In: GEOFFROY, J.J., MAURIÉS, J.-P; NGUYEN DUYJACQUEMIN, M. (eds.) Acta Myriapodologica. Mèm. Mus. natn. Hist. nat. 169: 523-532.

ADIS, J.; PAARMANN, W.; ERWIN, T.L. 1986. On the natural history and ecology of small terrestrial ground-beetles (Col.:Bembidiini: Tachyina: Polyderis) from an Amazonian black-water inundation forest. In: DEN BOER P.J.; LUFF M.L.; MOSSAKOWSKI D; WEBER F. (eds.) Carabid beetles. Their adaptations and dynamics. G. Fischer. Stuttgart, 
New York.p413-427.

ADIS, J.; PAARMANN, W.; HÖFER, H. 1990.

On phenology and life-cycle of Scarites sp. (Scaritini, Carabidae) from Central Amazonian floodplains. In: STORK N.E. (ed.) Ground beetles: their role in ecological and environmental studies. Intercept Ltda. Andover. p269-275.

BECK, L. 1969. Zum jahreszeitlichen Massenwechsel zweier Oribatidenarten (Acari) im neotropischen Überschwemmungswald. Verh. Dtsch. Zool. Ges., (Innsbruck 1968): 535-540.

...- 1972. Der Einfluß der jahresperiodischen Überflutungen auf den Massenwechsel der Bodenarthropoden im zentralamazonischen Regenwaldgebiet. Pedobiologia, 12: 133-148.

-1983. Bodenzoologie der amazonischen Überschwemmungswälder. Amazoniana, 8(1): 91-99.

CARICO, J. 1993. Revision of the genus Trechalea Thorell (Araneae, Trechaleidae) with a review of the taxonomy of the Trechaleidae and Pisauridae of the western hemisphere. $J$. Arachnol., 21: 226-257.

CARICO, J.; ADIS, J.; PENNY, N.D. 1985. A new species of Trechalea (Pisauridae: Araneae) from Central Amazonian inundation forests and notes on its natural history and ecology. Bull. Br. arachnol. Soc., 6(7): 289-294.

CHAPLEAU, F; JOHANSEN, P.H.; WILLIAMSON, M. 1988. The distinction between pattern and process in evolutionary biology: the use and abuse of the term 'strategy'. Oikos, 53(1); 136-138.

DISTER, E. 1985. Lebensräume und Retentionsfunktion. In: AKADEMIE FÜR NATURSCHUTZ UND LANDSCHAFTSPFLEGE (ed.) Die Zukunft der ostbayerischen Donaulandschaft. Laufener Seminarbeiträge 3/ 85. Laufen. 74-89.

-... 1988. Ökologie der mitteleuropäischen Auenwälder. In: WILHELMMÖNKER-STIFTUNG (ed.) Die Auenwälder gestern und heute - und morgen? No 4. Siegen. p6-30.
ERWIN, T.L. 1979. Thoughts on the evolutionary history of ground beetles: Hypothesis generated from comparative fauna analysis of lowland forest sites in temperate and tropical regions. In: ERWIN T.L.; BALL, G.E.; WHITEHEAD D.R.; HALPERN A.L. (eds.) Carabid beetles: Their evolution, natural history, and classification. W. Junk. The Hague. p539-592.

-..- 1981. Taxon pulses, vicariance, and dispersal: An evolutionary synthesis illustrated by carabid beetles. In: NELSON, G.; ROSEN, G. (eds.) Vicariance Biogeography. Columbia Univ. Press. New York. pl59-196.

ERWIN, T.L.; ADIS, J. 1982. Amazonian inundation forests. Their role as short-term refuges and generators of species richness and taxon pulses. In: PRANCE G.T. (ed.) Biological diversification in the tropics, Proc. V Int. Symp. Assoc. Trop. Biol. (Caracas 1979). Columbia Univ. Press. New York. p358-371.

FRIEBE, B.; ADIS, J. 1983. Entwicklungszyklen von Opiliones (Arachnida) im Schwarzwasser-Überschwemmungswald (Igapó) des Rio Tarumã Mirim (Zentralamazonien, Brasilien). Amazoniana . $8(1): 101-110$,

HÖFER, H. 1990. The spider community (Arachnida, Araneae) of a Central Amazonian blackwater inundation forest(Igapó). Acta Zool. Fenn., 190: 173-179.

IRMLER, U. 1979. Abundance fluctuations and habitat changes of soil beetles in Central Amazonian inundation forests (Coleoptera: Carabidae, Staphylinidae). Stud. Neotrop. Fauna Environ., 14: 1-16.

_._- 1981. Überlebensstrategien von Tieren im saisonal überfluteten amazonischen Überschwemmungswald. Zool. Anzeiger. 206(1/2): 26-38.

JUNK, W.J. 1989. Flood tolerance and tree distribution in Central Amazonian floodplains, In: HOLM-NIELSEN L.B.; NIELSEN I.C.; BALSLEV H. (eds.) Tropical forests - Botanical Dynamics, Speciation and Diversity. Academic 
Press. London. p47-64.

JUNK, W.J.; BAYLEY, P.B.; SPARKS, R.E. 1989.

The flood pulse concept in river-floodplain systems. Can. Spec. Publ. Fish. Aquat. Sci., 106: 110-127.

MARTIUS, C. 1989. Untersuchungen zur Okologie des Holzabbaus durch Termiten (Isoptera) in zentralamazonischen Überschwemmungswäldern (Värzea). AfraVerlag, Frankfurt am Main.285 p.

-- 1990. The influence of geophagous termites on soils of inundation forests in Amazonia - First results. In: VEERESH G.K.; MALLIK B.; VIRAKTAMATH C.A. (eds.) Social insects and the environment. New Dehli. p209-210.

.... 1992. Food provision storing by xylophagous termites in Amazonia (Isoptera; Nasutitermitidae). Entomol. Gener, 17(4); 296-276.

MESSNER, B.; ADIS, J. 1988. Die Plastronstrukturen der bisher einzigen submers lebenden Diplopodenart Gonographis adisi Hoffman 1985 (Pyrgodesmidae, Diplopoda). Zool. Jb. Anat. 117: 277-290.

MESSNER, B.; ADIS, J. 1992. Kutikuläre Wachsausscheidungenals plastronhaltende Strukturen bei Larven von Schaum- und Singzikaden (Auchenorrhyncha: Cercopidae und Cicadidae). Revue suisse Zool., 99(3): 713-720.

MESSNER, B.; ADIS, J.; RIBEIRO, E.F. 1992. Eine vergleichende Untersuchung über die Plastronstrukturen bei Milben (Acari). Dtsch. ent. Z. NF., 39(1-3): 159-176.

PAARMANN, W:; IRMLER, U.; ADIS, J. 1982. Pentacomia egregia Chaud. (Carabidae, Cicindelinae), an univoltine species in the Amazonian inundation forest. Coleopterists Bulletin, 36(2): 183-188.

PLATNICK, N.I.; HÖFER, H. 1990. Systematics and ecology of ground spiders (Araneae, Gnaphosidae) from Central Amazonian inundation forests. Am. Mus. Novitates, 2971: 1-16.

RIBEIRO, M. DE N.G.; ADIS, J. 1984. Local rainfall variability - a potential bias for bioeco- logical studies in the Central Amazon. Acta Amazonica, 14(1/2): 159-174.

SCHAEFER, M.; TISCHLER, W. 1983. Wörterbuch der Biologie: Ökologie. UTB, G. Fischer. Stuttgart. 354 p.

SOUTHWOOD, T.R.E. 1977. Habitat, the templet for ecological strategies? J. Anim. Ecol., 46; 337-365.

-.- -1988. Tactics, strategies and templets. Oikos, 52(1): 3-18.

TISCHLER, W. 1984. Einführung in die Ökologie. 3rd edit., G.Fischer. Stuttgart. 437 p.

TOMIUK, J.; VOHLAND, K.; BACHMANN, L.; ADIS, J. 1996. Ecological and genetic studies of the millipede Pycnotropis epiclysmus: Influence of flood water seasons on the evolution of ecotypes in tropical forests. Hereditas, 124: 298-299.

WALKER, I. 1992. Life history traits of shrimps (Decapoda: Palaemonidae) of Amazonian inland waters and their phylogenetic interpretation, In: ADIS J;; TANAKA S. (eds.) Symposium on life-history traits in tropical invertebrates. (INTECOL, Yokohama, Japan 1990). Studies on Neotropical Fauna and Environment, 27(2-3). Swets \& Zeitlinger. Lisse. p131-143.

WEST-EBERHARD, M.J. 1989. Phenotypic plasticity and the origins of diversity. Annu. Rev. Ecol. Syst, 20: 249-278.

WOLF, H.G.; ADIS, J. 1992. Genetic differentiation between populations of Neomachilellus scandens (Meinertellidae, Archaeognatha, Insecta) inhabiting neighbouring forests in Central Amazonia. Verh. naturwiss. Ver. Hamburg, (NF) 33: 5-13.

WORBES, M. 1986. Lebensbedingungen und Holzwachstum in zentralamazonischen Überschwemmungswäldern. Scripta Geobotanica, 17: 1-112.

-..- 1989. Growth rings, increment and age of trees in inundation forests, savannas and a mountain forest in the Neotropics. IAWA Bulletin N.S., 10(2); 109-122.

ZALESSKAJA, N.T. 1994. The centipede genus Lamyctes MEINERT, 1868, in the environs of Manaus, Central Amazonia, Brazil (Chilopoda, Lithobiomorpha, Henicopidae). Amazoniana, 13(1/2); 59-64.

Aceito para publicação em 25.09.96 